\title{
Generating isolated terawatt-attosecond x-ray pulses via a chirped-laser-enhanced high-gain free-electron laser
}

\author{
Zhen Wang, Chao Feng, ${ }^{*}$ and Zhentang Zhao \\ Shanghai Institute of Applied Physics, Chinese Academy of Sciences, Shanghai 201800, China
}

(Received 3 August 2016; published 4 April 2017)

\begin{abstract}
A feasible method is proposed to generate isolated attosecond terawatt $\mathrm{x}$-ray radiation pulses in highgain free-electron lasers. In the proposed scheme, a frequency chirped laser pulse is employed to generate a gradually varied spacing current enhancement of the electron beam, and a series of spatiotemporal shifters are applied between the undulator sections to amplify a chosen ultrashort radiation pulse from selfamplified spontaneous emission. Three-dimensional start-to-end simulations have been carried out, and the calculation results demonstrated that $0.15 \mathrm{~nm}$ x-ray pulses with a peak power over $1 \mathrm{TW}$ and a duration of several tens of attoseconds could be achieved by using the proposed technique.
\end{abstract}

DOI: 10.1103/PhysRevAccelBeams.20.040701

\section{INTRODUCTION}

The free-electron laser (FEL) is a device that utilizes relativistic electron beams as the gain medium to amplify the initial electromagnetic field. Unlike conventional lasers, the output photon energy range, temporal durations, and peak brightness of FELs are theoretically unlimited, which makes the FEL a unique and innovative approach for the realization of a tunable, high-intensity, coherent x-ray source. To date, several $x$-ray FEL facilities have been constructed worldwide [1-5] and have already enabled the observation and control of very fast phenomena at the atomic time scale, providing an ideal tool in various subjects such as femtochemistry, ultrahigh-resolution imaging, and the investigation of the dynamics in atomic and biological systems [6]. Nowadays, most of the existing and the under-construction x-ray FEL facilities [7-9] take advantage of the self-amplified spontaneous emission (SASE) scheme [10,11], which can provide a spatially coherent radiation pulse with a high peak power at the GW level and a pulse duration of about $100 \mathrm{fs}$. One of the major goals for future XFELs is to further enhance the peak power and shorten the pulse duration to increase the resolution of $\mathrm{x}$-ray diffraction imaging experiments and limit radiation damage on the samples simultaneously.

Several methods [12-19] have been developed in the past decade to manipulate the electron beam properties to generate ultrashort radiation pulses. A conceptually simple method is using a low-charge electron beam to reduce the

\footnotetext{
*Corresponding author.

fengchao@sinap.ac.cn

Published by the American Physical Society under the terms of the Creative Commons Attribution 4.0 International license. Further distribution of this work must maintain attribution to the author(s) and the published article's title, journal citation, and DOI.
}

electron bunch length as well as the radiation pulse length by about 2 orders of magnitude [12]. Alternately, one may adopt the emittance spoiler technique, which employs a slotted foil in the central of the bunch compressor to select a small unspoiled part of the electron beam to lase [13]. Another concept, dubbed enhanced SASE (ESASE), takes advantage of an external few-cycle laser to manipulate the electron beam longitudinal phase space to enhance the peak current in a short slice for generating attosecond radiation pulses [14]. The low-charge operation and the emittance spoiler technique have been experimentally demonstrated at the linac coherent light source (LCLS) to generate hard $\mathrm{x}$-ray pulses with a few femtosecond pulse durations $[12,15]$. There is also a plan for developing the ESASE technique at the LCLS in the near future [16]. However, none of these techniques hold the ability to enhance the output peak power beyond saturation, and the shortest output pulse length from ESASE is limited by the FEL slippage length, which is on the order of a few hundred attoseconds.

In order to overcome the FEL pulse lengthening from the slippage effect, the mode-locking concept has been applied to SASE FELs for generating attosecond pulse trains. In the initial proposed scheme [17], a periodic variation in the electron beam energy or density is introduced by a highpower optical laser; then a mode-locked undulator, which consists of a series of undulator-chicane modules, is imposed to add precise delays between the electron beam and the radiation field and thereby continually amplify the ultrashort x-ray pulse trains. Recently, a pulse-compression scheme [18] that combines the ESASE principle and the mode-locking technique has been proposed to achieve an up to hundredfold increase in the peak power of x-ray pulses with a pulse duration of 50 as and photon energies around $10 \mathrm{keV}$. However, an additional x-ray delay module makes the layout technically very challenging. Later on, an alternative method [19] based on the emittance spoiler 
technique is proposed to simplify the overall design. A multiple-slotted foil with uneven spacing of slots has been adopted to prevent the buildup of multiple pulses and allows one to amplify only one single short pulse by properly setting the strengths of delay sections. However, in this scheme, dominated by the uncorrelated energy spread and betatron beam size, it will be very challenging to generate subfemtosecond radiation pulses [15].

In this paper, we propose a novel and feasible scheme to generate isolated terawatt $\mathrm{x}$-ray pulses with pulse durations of several tens of attoseconds based on the ESASE and superradiant principles [20,21]. However, no x-ray delay sections are required, which makes the proposed technique much simpler and easily applied to existing and future $\mathrm{x}$-ray FELs. When compared with the emittance spoiler technique, the proposed technique holds the ability of achieving shorter output pulse durations and has the additional advantage of natural synchronization to external lasers, which is especially important for pump-probe experiments.

The paper is organized as follows: the principle of the proposed technique is described in Sec. II. Then an optimized design for a hard $\mathrm{x}$-ray FEL based on the proposed scheme is given and three-dimensional start-toend simulations have been performed in Sec. III. Finally, we conclude in Sec. IV.

\section{PRINCIPLES OF THE PROPOSED TECHNIQUE}

The schematic layout of the proposed technique is illustrated in Fig. 1, consisting of an ESASE section upstream of the undulator and some delay lines inserted among the undulator sections. In the conventional ESASE scheme, an ultrashort laser pulse with no more than two optical cycles is employed to interact with the electron beam in a single period wiggler to introduce an energy modulation. Then the electron beam is sent through a dispersive magnetic chicane to convert the energy modulation into density modulation, which results in an isolated current enhancement along the beam. This kind of fewcycle laser can be achieved through the chirped pulse amplification technique (CPA) [22], where a longitudinal stretched, frequency chirped laser pulse is amplified by the gain medium and sent through an optical element (compressor) with a suitable chromatic dispersion to recompress the beam to a bandwidth-limited pulse. The main challenge in the pulse compression process lies in effective dispersion compensation and hence compression of the generated bandwidths to yield an isolated ultrashort optical pulse.

In the proposed scheme, instead of a few-cycle laser pulse, a frequency chirped laser is utilized to imprint a gradually varied spacing current enhancement on the electron beam. This powerful laser pulse with significant and adjustable frequency chirp can be easily obtained by tuning the parameters of the compressor of the CPA. The dispersion in the laser pulse does not need to be fully compensated, which would significantly simplify the laser system design for the ESASE scheme. After the density modulation, the electron beam is sent into a long undulator with a series of spatiotemporal shifters between the undulators to amplify a chosen ultrashort radiation pulse via the superradiant gain process. In the first undulator section, the gradually varied spacing current enhancement leads to an uneven spacing radiation pulse train. The first undulator should be relatively long to amplify the target radiation pulse with a peak power that is high enough to suppress the shot noise in the following undulator section but still far from saturation to prevent degradation of the electron beam quality. The target radiation pulse is then shifted forward to the following current peak by the delay line, while the other radiation pulses are shifted to the lowcurrent part of the beam. The microbunching formed in the current peaks is smeared out by the delay line, preventing the continuous growth of noisy spikes in the following undulators. The target radiation pulse reseeds the fresh peak in the following undulator section, leading to a continuing amplification of the radiation pulse. Repeating this process in the following undulator sections, the target ultrashort pulse can be finally amplified to the terawatt level.

\section{3D SIMULATIONS FOR THE PROPOSED TECHNIQUE}

To illustrate the physical mechanism and a possible application with realistic parameters, three-dimensional start-to-end simulations have been performed for the proposed technique. We take the layout and nominal parameters of the Shanghai hard x-ray FEL facility (HXFEL) [23], which is aimed at generating a GW-level femtosecond radiation pulse based on SASE. The linac of the HXFEL consists of an $S$-band photoinjector, a laser heater system, an

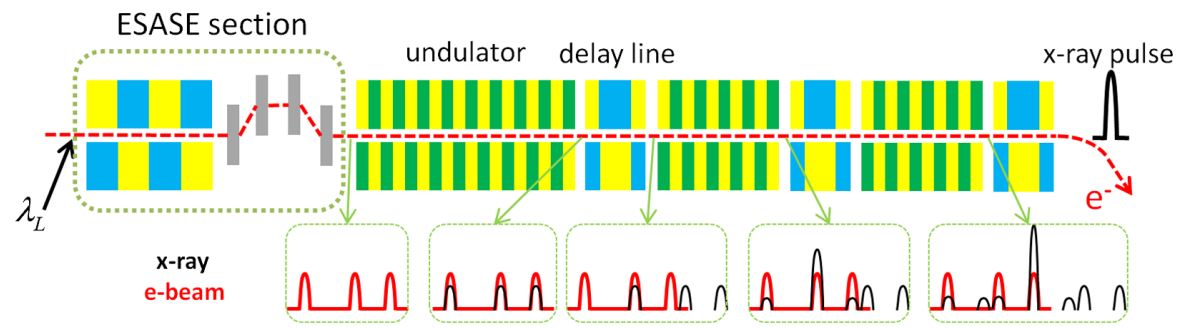

FIG. 1. Schematic layout of the proposed technique. 

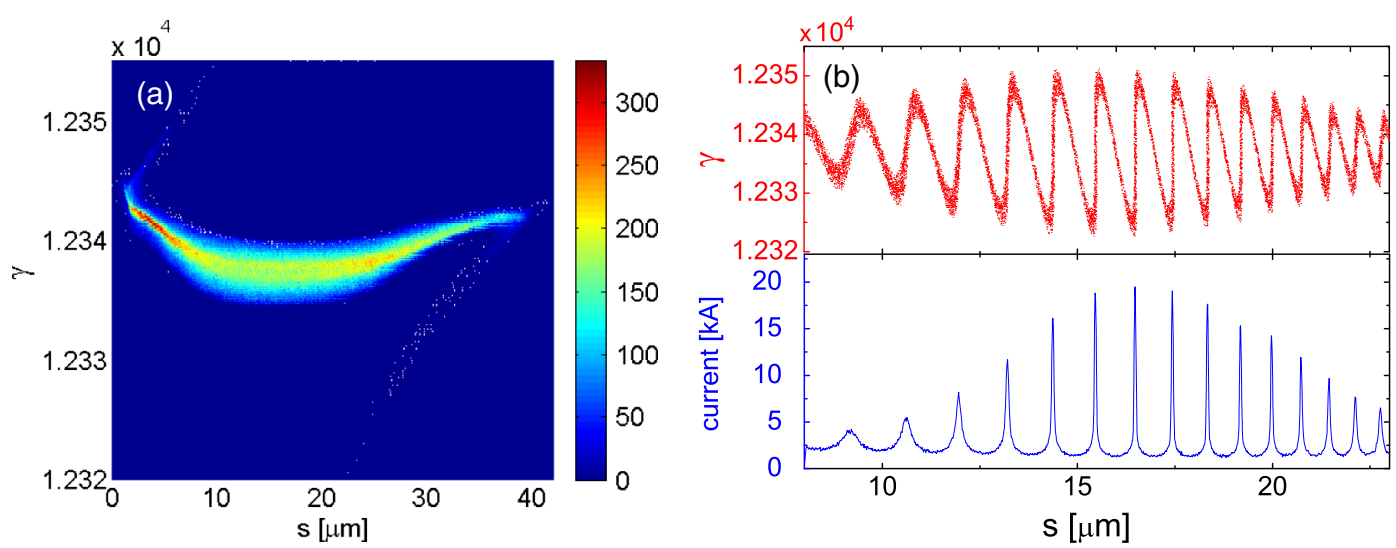

FIG. 2. (a) Longitudinal phase space at the end of the linac. (b) Longitudinal phase space and the corresponding current distribution at the end of the ESASE section. The bunch head is to the right.

$X$-band linearizer, two bunch compressors, and three $C$-band main accelerator sections. At the exit of the linac, the electron beam can be accelerated to about $6.3 \mathrm{GeV}$ with the charge of $250 \mathrm{pC}$ and peak current of $3 \mathrm{kA}$. ASTRA [24] and ELEGANT [25] were used for the simulations in the photoinjector and the remainder of the linac, respectively. The longitudinal phase space of the electron beam at the end of the linac is shown in Fig. 2(a), where one can find that a constant profile of the beam energy, energy spread, and emittance is maintained in an approximately $50 \mathrm{fs}$ region in longitudinal. In this region, the normalized transverse emittances are around $0.4 \mathrm{~mm}-$ mrad in both the horizontal and vertical directions, and the slice energy spread is about $600 \mathrm{keV}$. Simulations for the modulation process of ESASE were done with a threedimensional algorithm [26] based on the fundamentals of electrodynamics when considering the appearance of electric and magnet fields of a chirped laser beam. The FEL gain processes were simulated by GENESIS [27]. A chirped laser pulse with the central wavelength of $800 \mathrm{~nm}$, peak power of about $80 \mathrm{GW}$, beam waist of $200 \mu \mathrm{m}$, and pulse length of $40 \mathrm{fs}$ (FWHM) is injected to the ESASE modulator (a single period wiggler with a period length of $20 \mathrm{~cm}$ ). The bandwidth of the laser pulse is about $34 \%$, equivalent to a 3.5 fs transform-limit pulse after full pulse compression.

The longitudinal phase space and current distributions of the electron beam at the exit of the ESASE section are shown in Fig. 2(b), where a gradually varied spacing current enhancement is achieved with the peak current of about $20 \mathrm{kA}$. The energy modulation amplitude induced by the laser pulse is about 10 times the initial beam energy spread. The duration of the current spikes in the vicinity of the beam center is on the scale of $\sim 100$ as. This electron beam is then sent into the following undulator sections to generate ultrashort radiation pulses at $1.5 \AA$. The simulation results for the $\mathrm{x}$-ray radiation profile evolution in the undulator are illustrated in Fig. 3.

The length of each undulator segment is about $2 \mathrm{~m}$ (100 periods) with a period length of $18 \mathrm{~mm}$. The undulator segments in the 1st-10th undulator sections are chosen to be $7,4,3,2,2,2,2,2,2$, and 2 , respectively, to optimize the FEL power and improve the contrast of the target pulse against others. The first undulator section with seven segments was used to produce an attosecond pulse train with hundred-megawatt-level peak power. The longitudinal profile of the radiation reflects the comblike structure of the current distribution, as shown in Fig. 3(a). After that, delay lines are applied to selectively amplify the target pulse (indicated in red) in the following undulator segments. The delay distances induced by these delay lines should be also gradually varied to exactly match the interspacings between the current peaks. In order to realize this in a real machine, one needs to measure the frequency distribution of the chirped laser by using optical techniques, providing a reference for setting the strengths of the delay sections. And then one should scan the strengths of these delay sections separately to optimize the final output intensity.

In the second undulator section, the target radiation pulse was shifted forward and overlapped with the next current peak, leading to a continual power amplification and pulse shortening due to the superradiant behavior of the ultrashort radiation pulse [20,21]. Beside the target radiation pulse, we found in the simulations that there is still some degree of overlap between other radiation pulses and current peaks due to the relatively long radiation pulse length (long slippage length) generated from the first undulator section. This results in the appearance of several satellite pulses in addition to the target pulse, as shown in Fig. 3(b). However, the amplification of these satellite pulses does not proceed as the slippage length becomes shorter and shorter in the following undulator sections. As shown in Fig. 3(d), after five undulator sections, the peak power of the target radiation pulse is enhanced to about $500 \mathrm{GW}$, which is already 2 orders of magnitude higher than that of the satellite pulses. The contrast, which is defined by the percentage of the energy contained in the target pulse, is further improved in the following undulators. After ten undulator sections, an isolated radiation pulse with a pulse length of about 80 as (FWHM) and a peak power of about 

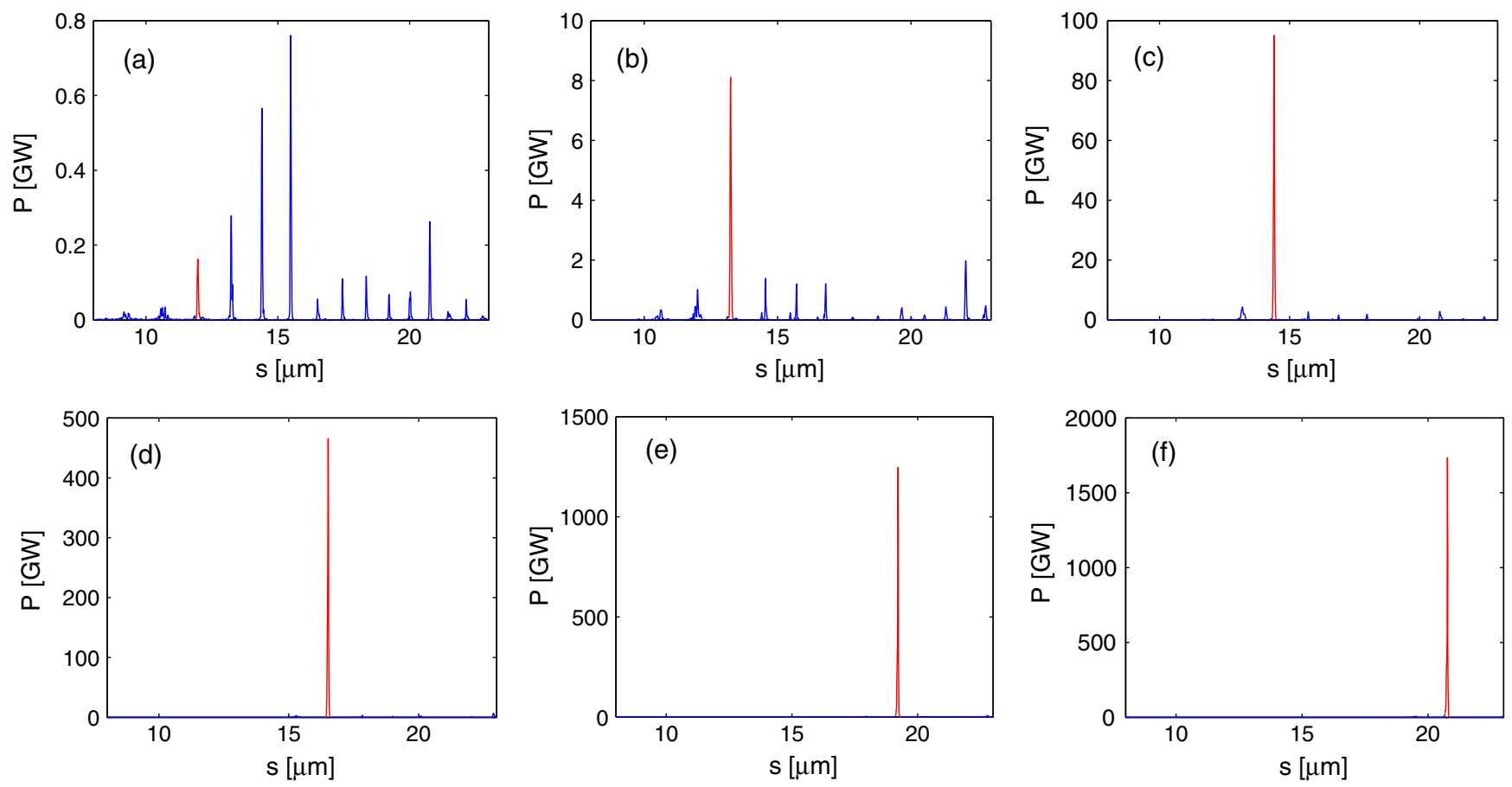

FIG. 3. XFEL radiation structure evolutions along the undulator beam line at the end of (a) the 1st, (b) the 2nd, (c) the 3rd, (d) the 5th, (e) the 8 th, and (f) the 10th undulator section.

1.7 TW is produced, as shown in Fig. 3(f), while the other (mainly "satellite") pulses are still at a few-GW level. The contrast of the target radiation pulse is over $96 \%$. The final output power can be further increased by utilizing more undulator sections and more current spikes. It is worth pointing out that the GW-level satellite pulses in the final output may still cause potential difficulty on user experiments and applications in the future.

Figure 4 shows the FEL peak power growths along the undulator distance. For comparison purposes, simulations were also performed for the normal SASE case (blue line) with the same electron beam from the linac and the same undulators but without delay lines. The normal SASE FEL gets saturation at around $45 \mathrm{~m}$ of the undulator with the saturation power of about $13 \mathrm{GW}$. According to the basic

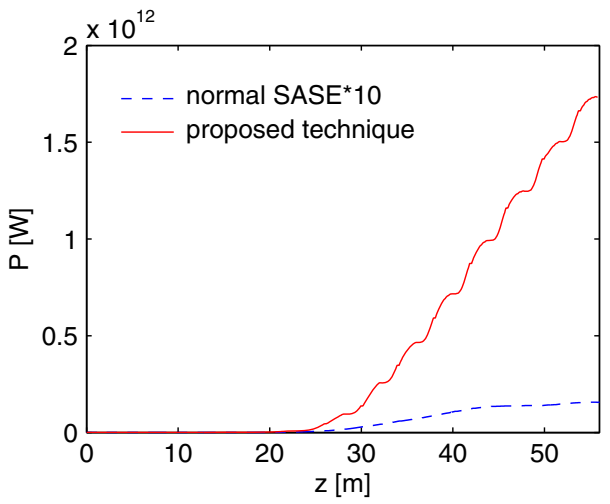

FIG. 4. FEL peak power gain curves for normal SASE (blue dashed line) and the proposed scheme (red solid line). theory of a high-gain FEL, the saturation power is proportional to $I_{e}^{4 / 3}$, where $I_{e}$ is the peak current $[28,29]$. In the proposed scheme, the peak current has been enhanced by over 6 times (from 3 to about $20 \mathrm{kA}$ ) through ESASE, leading to about 10 times enhancement of the saturation power in each undulator section. In addition, about ten pulses are compressed to a single radiation pulse. Thus, the final output peak power is about 2 orders of magnitude higher (red line) than the normal SASE. From Fig. 4, one can also find that the peak power for the proposed scheme grows quadratically with the undulator length in each undulation section after the third undulator section. The strong seed generated from previous undulators initiates this superradiant process, which helps the ultrashort radiation pulse quickly extract energy from the electron beam in a relatively short undulator section.

The final output pulse duration of the proposed scheme is determined by the widths of the current spikes and the slippage lengths in the last few undulator sections, where the FEL power gain is quite large. For a given energy modulation amplitude, the width of the current spike is mainly determined by the initial uncorrelated energy spread of the electron beam, which can be tuned by changing the bunch compression factor of the linac. Here we performed simulations with the same parameters used above but an electron beam with a peak current of $1 \mathrm{kA}$ and an initial energy spread of about $200 \mathrm{keV}$. The simulation results are summarized in Fig. 5. Although the durations of the current peaks have been reduced by about 3 times, the final output pulse duration is reduced only by about 1.5 times, from 80 

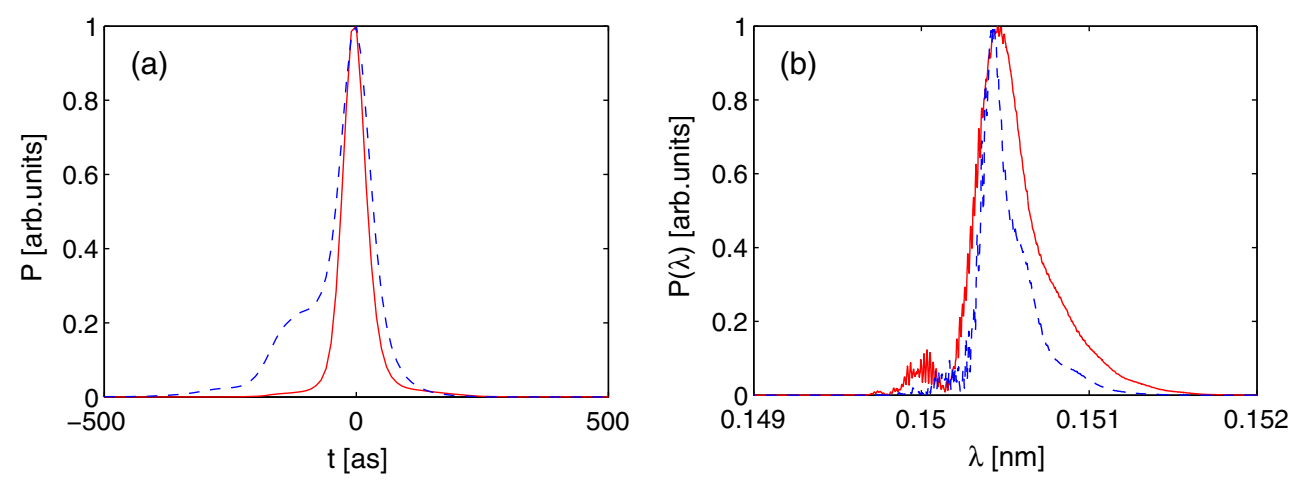

FIG. 5. Comparisons of the single shot radiation pulse (a) and corresponding spectra (b) from the proposed scheme with an initial electron beam peak current of 3 (blue dashed line) and $1 \mathrm{kA}$ (red solid line).

to about 52 as, as shown in Fig. 5(a). The minimal output pulse duration is limited by the FEL slippage length, which is about 100 as in our case. One can further reduce the output pulse length by using shorter undulator sections; however, the FEL gain will be significantly degraded. The contrast of the target radiation pulse against the background for this case is over $98 \%$, which results in a quite clear output spectrum, as shown in Fig. 5(b). The FWHM bandwidth of the spectrum is about $0.2 \%$, which is about 1.5 times wider than the Fourier transform limit.

In the above simulations, the required density modulation is introduced by a chirped laser with a very broad bandwidth, which may limit the applications of the proposed technique. We found later that the task can also be performed by utilizing a commercial laser with a central wavelength of $800 \mathrm{~nm}$ and a spectral bandwidth of about $17 \%$ (equivalent to a $7 \mathrm{fs}$ transform-limit pulse). However, as the separation differences between adjacent current peaks become smaller, the lengths of the first few undulator sections should be reduced to prevent the power growth of satellite pulses. According to our simulations, the numbers of undulator segments in each undulator section are chosen to be $6,3,2,2,2,2,2,2,2$, and 2 to optimize the contrast. An isolated $x$-ray radiation pulse with a pulse duration of around 80 as, peak power of around $1 \mathrm{TW}$, and contrast of about $85 \%$ can be generated at the exit of a $50 \mathrm{~m}$ long undulator.

\section{CONCLUSIONS}

In conclusion, a novel and easy-to-implement method has been proposed to significantly improve the output properties of a SASE FEL. By making modifications of the laser system of ESASE and adding small delay lines between undulator sections, terawatt-attosecond isolated radiation pulses could be produced. Three-dimensional start-to-end simulations have been carried out to show the possible performance of the proposed technique with realistic parameters of a hard x-ray FEL facility, and the simulation results demonstrated that a fully coherent hard $\mathrm{x}$-ray radiation pulse with a peak power at the terawatt level and a pulse duration less than 100 as can be achieved in a $\sim 50 \mathrm{~m}$ undulator. It is worth pointing out that the parameters used in our simulations are representative rather than fully optimized design sets. The output peak power can be further enhanced by using a longer chirped laser (more current spikes), more undulator sections, and better beam parameters, e.g., higher beam energy or smaller initial energy spread. So we believe that the proposed scheme can be easily applied to the current existing and future x-ray FEL facilities to generate a multiterawatt FEL pulse. This kind of coherent x-ray light source is potentially useful where ultrabrightness and ultrashort pulses are required. It may open a new regime of ultrafast x-ray sciences, such as $\mathrm{x}$-ray nonlinear optics and $3 \mathrm{D}$ atomic-resolution imaging of single molecules [30,31].

\section{ACKNOWLEDGMENTS}

The authors thank B. Liu, H.X. Deng, Q. Gu, and D. Wang for helpful discussions and useful comments. This work is supported by the National Natural Science Foundation of China (11475250) and Youth Innovation Promotion Association CAS.

Note added in proof.- - It has been brought to our attention after the acceptance of this article that the following related article [32] has also appeared. However, in our paper, we have demonstrated with three-dimensional start-to-end simulations and realistic parameters that the proposed technique can be realized by using a commercial laser at $800 \mathrm{~nm}$. We also show the optimization method for the proposed technique and study the effects of electron beam compression factor, initial laser pulse length and FEL slippage on the final output radiation pulse length.

[1] W. A. Ackermann et al., Operation of a free-electron laser from the extreme ultraviolet to the water window, Nat. Photonics 1, 336 (2007). 
[2] P. Emma et al., First lasing and operation of an ångstromwavelength free-electron laser, Nat. Photonics 4, 641 (2010).

[3] T. Ishikawa et al., A compact X-ray free-electron laser emitting in the sub-ångstrom region, Nat. Photonics 6, 540 (2012).

[4] E. Allaria et al., Two-stage seeded soft-X-ray free-electron laser, Nat. Photonics 7, 913 (2013).

[5] C. Pellegrini, A. Marinelli, and S. Reiche, The physics of X-ray free-electron lasers, Rev. Mod. Phys. 88, 015006 (2016).

[6] C. Bostedt, S. Boutet, D. M. Fritz, Z. Huang, H. J. Lee, H. T. Lemke, A. Robert, W. F. Schlotter, J. J. Turner, and G. J. Williams, Linac Coherent Light Source: The first five years, Rev. Mod. Phys. 88, 015007 (2016).

[7] M. Altarelli et al., DESY Technical Design Report No. DESY-2006-097, 2007.

[8] R. Ganter, PSI Report No. PSI-10-04, 2010.

[9] J. H. Han, H. S. Kang, and I. S. Ko, Status of the Pal-XFEL project, in Proceedings of the 3rd International Particle Accelerator Conference, New Orleans, LA, 2012 (IEEE, Piscataway, NJ, 2012), p. 1735.

[10] A. Kondratenko and E. Saldin, Generation of coherent radiation by a relativistic-electron beam in an undulator, Part. Accel. 10, 207 (1980).

[11] R. Bonifacio, C. Pellegrini, and L. M. Narducci, Collective instabilities and high-gain regime in a free electron laser, Opt. Commun. 50, 373 (1984).

[12] Y. Ding et al., Measurements and Simulations of Ultralow Emittance and Ultrashort Electron Beams in the Linac Coherent Light Source, Phys. Rev. Lett. 102, 254801 (2009).

[13] P. Emma, K. Bane, M. Cornacchia, Z. Huang, H. Schlarb, G. Stupakov, and D. Walz, Femtosecond and Subfemtosecond X-Ray Pulses from a Self-Amplified SpontaneousEmission-Based Free-Electron Laser, Phys. Rev. Lett. 92, 074801 (2004).

[14] A. A. Zholents, Method of an enhanced self-amplified spontaneous emission for x-ray free electron lasers, Phys. Rev. ST Accel. Beams 8, 040701 (2005).

[15] Y. Ding et al., Femtosecond X-Ray Pulse Characterization in Free-Electron Lasers Using a Cross-Correlation Technique, Phys. Rev. Lett. 109, 254802 (2012).

[16] Z. Huang, in Workshop on Tailored Soft X-Ray Pulses (PSI, Switzerland, 2016).

[17] N. R. Thompson and B. W. J. McNeil, Mode Locking in a Free-Electron Laser Amplifier, Phys. Rev. Lett. 100, 203901 (2008).
[18] T. Tanaka, Proposal for a Pulse-Compression Scheme in X-Ray Free-Electron Lasers to Generate a Multiterawatt, Attosecond X-Ray Pulse, Phys. Rev. Lett. 110, 084801 (2013).

[19] E. Prat and S. Reiche, Simple Method to Generate Terawatt-Attosecond X-Ray Free-Electron-Laser Pulses, Phys. Rev. Lett. 114, 244801 (2015).

[20] R. Bonifacio, L. De Salvo Souza, P. Pierini, and N. Piovella, The superradiant regime of a FEL: Analytical and numerical results, Nucl. Instrum. Methods Phys. Res., Sect. A 296, 358 (1990).

[21] R. Bonifacio, N. Piovella, and B. W. J. McNeil, Superradiant evolution of radiation pulses in a free-electron laser, Phys. Rev. A 44, R3441 (1991).

[22] P. Maine, D. Strickland, P. Bado, M. Pessot, and G. Mourou, Generation of ultrahigh peak power pulses by chirped pulse amplification, IEEE J. Quantum Electron. 24, 398 (1988).

[23] Z. T. Zhao et al., Design of a compact x-ray free electron laser, in Proceedings of the 32nd Free Electron Laser Conference, Malmö, Sweden (Max-lab, Sweden, 2010), p. 626.

[24] K. Floettmann, ASTRA User's Manual, available at http:// www.desy.de/mpyflo/Astra_dokumentationS.

[25] M. Borland, Simple method for particle tracking with coherent synchrotron radiation, Phys. Rev. ST Accel. Beams 4, 070701 (2001).

[26] H. Deng, T. Lin, J. Yan, D. Wang, and Z. Dai, Threedimensional numerical investigations of the laser-beam interactions in an undulator, Chin. Phys. C 35, 308 (2011).

[27] S. Reiche, GENESIS 1.3: A fully 3D time-dependent FEL simulation code, Nucl. Instrum. Methods Phys. Res., Sect. A 429, 243 (1999).

[28] M. Xie, Exact and variational solutions of 3D eigenmodes in high gain FELs, Nucl. Instrum. Methods Phys. Res., Sect. A 445, 59 (2000).

[29] Z. Huang and K. J. Kim, Review of x-ray free-electron laser theory, Phys. Rev. ST Accel. Beams 10, 034801 (2007).

[30] M. Fuchs et al., Anomalous nonlinear X-ray Compton scattering, Nat. Phys. 11, 964 (2015).

[31] A. Aquila et al., The linac coherent light source single particle imaging road map, Struct. Dyn. 2, 041701 (2015).

[32] T. Tanaka et al., Using irregularly spaced current peaks to generate an isolated attosecond X-ray pulse in free-electron lasers, J. Synchrotron Rad. 23, 1273 (2016). 